\title{
Lane-level Path Guiding Method for Unmanned Ground Vehicle
}

\author{
Qiangrong Yang ${ }^{1^{*}}$, Jianyao Hu${ }^{1}$, Meiling Wang ${ }^{2}$, Huachao Yu ${ }^{2}$ and Qi Peng ${ }^{1}$ \\ ${ }^{1}$ The Fifth Research Institute of MIIT, Guangzhou, China, 510610 \\ ${ }^{2}$ Beijing Institute of Technology, Beijing, China, 100081 \\ *corresponding author email:bit20070042
}

Keywords: Intelligent Robot, Unmanned ground vehicle, Lane-level, Digital map, Path guiding.

\begin{abstract}
A lane-level digital map is constructed with multi-sensors to meet the requirements of unmanned ground vehicle for high-precision digital map and the needs to navigate in structured road environment. Based on this lane-level digital map, a lane-level path guiding algorithm is also proposed. First, a lane-level digital map is constructed with the multi-sensor data collected by data collecting platform and the lanes and segments are built according to the traffic rules. Next, different property tables are designed for different geographic elements to represent their characteristics. Then, a global path planning method is proposed according to the characteristic of structured road environment. Finally, a local path planning method is proposed to avoid the obstacles when UGV meets the obstacles. According to the results of on-vehicle experiments and contests for unmanned vehicles, the proposed digital map and path guiding method prove to meet the requirements of unmanned ground vehicles in structured road environment and offer reliable guidance and guarantee for fast and safe driving of unmanned ground vehicles.
\end{abstract}

\section{Introduction}

Unmanned ground vehicle (UGV) is the main target of future transportation and combat system researches, the results of which will make significant differences for human society. Recently, a requirement of the vehicle-mounted digital map to be more precise has been made with the application of UGV in ITS (intelligent transportation system), ADAS (advanced driver assistant system), intelligent transportation. For the above reasons, the related researches of lane-level digital map and lane-level path guiding have become the focus of many researchers, such as the construction of lane-level digital map[1]-[3], road modelling[4], localization based on prior map information[5] and map matching based on prior map[6].

In this paper, a lane-level digital map for UGV is constructed with the data fusion of multisensors and a lane-level path guiding method is proposed based on this high-precision map, which will offer reliable guidance and guarantee for fast and safe driving of unmanned ground vehicles in unstructured environment. 


\section{Data Collecting and Processing}

\subsection{Data Collecting Platform}

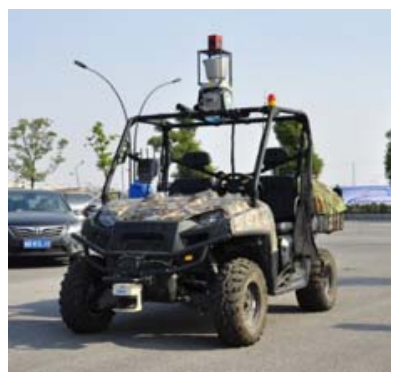

Figure 1. Data collecting platform $\mathrm{IN}^{2} \mathrm{BOT}$.

The UGV platform IN2BOT in Figure 1 is chosen as the data collecting platform and it's modified from the US all-terrain vehicles Polaris. The gear, throttle and brakes on IN2BOT can be controlled automatically. Moreover, IN2BOT is mounted with all the necessary sensors for building lane-level digital map, which include positioning sensors, panoramic cameras, cameras for traffic sign and traffic light, LIDAR. The mounted sensors are described in table 1.

Table 1. Sensors on IN2BOT.

\begin{tabular}{|c|c|c|}
\hline Sensor & $\begin{array}{l}\text { Mounting } \\
\text { position }\end{array}$ & Function \\
\hline 3D Lidar & Top & Recognizing the obstacles \\
\hline 2D Lidar & Front/Back & Cover the blind zone of 3D Lidar \\
\hline Millimetre-wave radar & Front & Capturing the dynamic information \\
\hline Panoramic camera & Top & Receiving the vision information of the environment \\
\hline Monocular cameras & Front & Recognizing the traffic sign and traffic light \\
\hline Position system & Top & Positioning of vehicle \\
\hline
\end{tabular}

\subsection{Data collecting Solution}

A data collecting solution is proposed to meet the requirements of different geographic data and the traditional sensor configuration of data collecting platform for map building. The data collecting solution consist of the following four parts:

(1) Collecting the panoramic images. The panoramic images provide the basic information for the high-precision map and the collecting process is as follows: First, the panoramic camera is calibrated with the designed calibrating board shown in Figure 2. Then, the top-view image of the environment is achieved from the original image with inverse projection mapping (IPM) method. Finally, a QR code is placed in every image to infer the position of the vehicle where the image is taken, which will be an essential information for the following image-fusing process. The collecting process of panoramic images is shown in Figure 3.

(2) Collecting the positioning data. The positioning data for building lane-level digital map should be precise enough to distinguish different lanes, which means the accuracy of the positioning data is less than 1 decimeter. However, the accuracy of the widely-applied GPS is larger than 1 meter. To solve the above problem, the differential positioning technology is used in this paper to improve the accuracy of the positioning system.

(3) Estimating the position of traffic light and traffic sign. A multi-object tracking and positioning algorithm in [7] is utilized to estimate the position of traffic light and traffic sign after receiving the images from the monocular cameras.

(4) Determining the road edge. A multi-characteristic Hough transformation is used on LIDAR data from the environment to determine the road edge. 


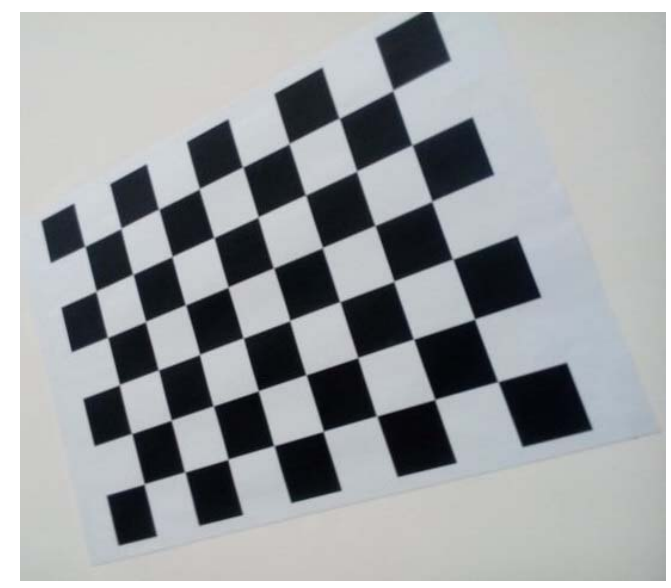

Figure 2. The designed calibrating board.

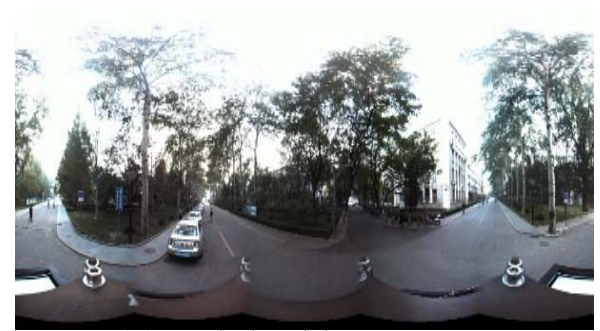

(a) Original image

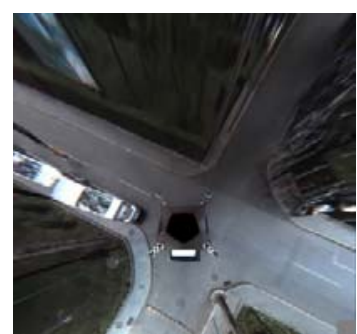

(b) top-view image

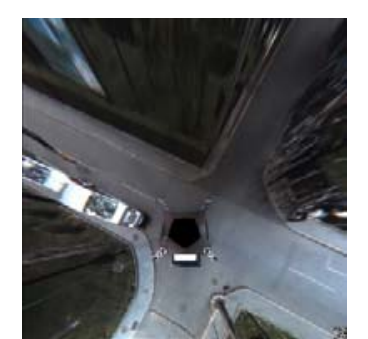

(c) image with QR code

Figure 3. Collecting process of panoramic images.

\section{Lane-level Digital Map}

\subsection{Building the Digital Map}

In this section, a lane-level digital map is built with panoramic image sequences. First, the region where the panoramic image sequences are collected are transformed into the image coordinate system shown in Figure 4 due to the large area and huge data. In this coordinate system, the size of a grid is $1620 \mathrm{~m} \times 360 \mathrm{~m}(27000$ pixels $\times 6000$ pixels $)$ and the origin of the coordinate system $\left(x_{0}, y_{0}\right)$ is chosen according to the range of the image collecting region.

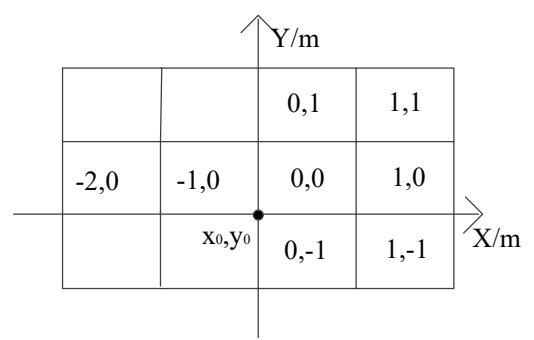

Figure 4. Designed coordinate system.

When a new image is captured, the image is transformed into the designed coordinate system with the direction of vehicle $\varphi_{i}$ and the position of the vehicle $\left(x_{i}, y_{i}\right)$ which is stored in the image. Then, the grid number of current image $(i, j)$ is calculated with the position of the vehicle and the coordinate of the origin and determines if the grid number of the former image is equal to $(i, j)$. If these two images are in the same grid, merge these two images according to their stored positions. Otherwise, save the former image and check the database that if there is any stored images in the grid $(i, j)$. If there is any images in the grid $(i, j)$, merge these images and current image. Otherwise, store current image into grid $(i, j)$. Finally, repeat this process until all the images are merged and merged all the images in different grids into one large image. 
After getting the huge image of the data collecting region, the digital map can be built with traffic rules and turning restriction for different lanes. For roads with 4 or more lanes, the turning property of different lanes are usually different. For instance, the left lane is forbidden to turn right and the right lane is forbidden to turn left. Since the connection between different roads should be built according the above turning restrictions. Moreover, it's necessary to collect different lanes of the same road to ensure that the vehicles are driving in the right lane according to the traffic rules when driving through the intersections.

\subsection{Road Property Table}

Except for the precise position of geographic objects, the digital map should also provide precise property for each geographic objects to reveal the geographic characteristic of them. For the above reason, the road property table is constructed in this section to meet the requirements of UGV.

The property table for line dataset "road" is shown in table II. The property "Left_Width" and "Right_Width" represents the distance between the vehicle and the left border or right border of the road. These two properties give the left and right margin for the local planning of UGV, which guarantees the safety of UGV to drive through the roads. These two properties are calculated through the road edge data. The property "Speed_Limit" represents the speed limit of the road, which enables UGV to drive according to the traffic rules. Moreover, some speed limits are set to the roads which are near the intersection to ensure the safety of UGV (for instance, the speed limit of the lanes to turn left or right is set to be $15 \mathrm{~km} / \mathrm{h}$ and the speed limit of other lanes is set to be 30 $\mathrm{km} / \mathrm{h}$ ). The property "Change_Direction" represents the turning restriction of the lane (0: go ahead, 1: turn left, 2: turn right, 3: turn round). Property "if_CrossLine" represents that if the lane is allowed to take over (0: forbidden, 1: allowed). In some specific road regions, the vehicles are forbidden to take over and since this property offers reliable guidance for UGV to drive through these regions. Property "Direction" represents the direction of the road, which is represented by the heading angle of the road.

Table 2. Property for line dataset Road.

\begin{tabular}{cccc}
\hline Property & Data type & Default & meaning \\
\hline Left_Width & double & 0.0 & Distance to the left border \\
Right_Width & double & 0.0 & Distance to the right border \\
Speed_Limit & double & 0.0 & Speed limit of road \\
Change_Direction & int & 0 & Turning restriction of road \\
if_CrossLine & int & 0 & Take-over property \\
Direction & double & 0.0 & Direction of road \\
\hline
\end{tabular}

\section{Path Guiding}

The typical scene for UGV is that the vehicle uses path planning method to get the global optimal path according to the given task point sequence and modify the global path with local planning method and real-time information from the sensors, which enables the UGV to get to the Finish point safely and quickly. From the above scene, it's essential for UGV to solve the problems that how to get the global optimal path with the given task point sequence and how to modify the global path with the information from the sensors, which will be discussed in the following section.

\subsection{Global Path Planning}

The lane-level global path planning algorithm mainly consists of the following steps:

1) Road network abstraction. In this step, the roads are abstracted as line segments and the intersection is abstracted as a point. In this way, the complex urban road network is abstracted as a directed-weighted network $G(V, L)$, where $V$ and $L$ are set of points and line segments respectively, and $W$ is the weight matrix for line segments set $L$.

2) Map matching of the task points. Since the precision of the task points is relatively low, the 
task point will be matched to a wrong road if the traditional map matching method is directed applied. To solve the above problem, a map matching method integrating the property of the task point and the lane is proposed in this step. First, a circle with the task point as the centre is drawn as the buffer region and all the roads which are intersected with the buffer region are chosen as the candidate roads. Then, determine the direction of the current task point with the former task point and delete all the roads whose directions are quite different from the direction of the current task point. Next, match the current task point to the corresponding lane according to the turning property of the task point. Finally, the node of the matched lane is added to the key point set $V_{L}$.

3) Determining the weight matrix $\boldsymbol{W}$. According to the chosen global path planning method below, the weight of the road is set to be the length of the road:

$$
w_{i, j}=l_{i . j}
$$

where $w_{i, j}$ is the weight of the line segment $<i, j>$, and $l_{i, j}$ is the length of line segment $<i, j>$, and if $<i, j>\notin L$, then $l_{i . j}=\infty$.

4) Global path planning. Since the number of the nodes in the urban road network is very huge and every task point must be passed by the global optimal path, the global searching will be calculated $n-1$ times by the traditional path planning method to get the global optimal path ( $n$ is the number of nodes in the key point set $V_{L}$ ), which will cost unreasonable computation time. To solve the above problem, the searching region of the algorithm in this paper is restricted to the region that the sum of the distance between the point and the start point and the distance between the point and the finishing point is less than $2 d_{s, e}\left(d_{s, e}\right.$ is the distance between the start point $\mathrm{s}$ and the finishing point e). All the nodes in the restricted region are selected into the searching set $V_{s}$ and the global optimal path is determined as follows: first, let $\operatorname{dist}(s)=0, \operatorname{dist}(i)=\infty\left(i \neq s\right.$ and $\left.i \in V_{S}\right)$. Then, choose one node $u$ from set $V_{s}$ which meets the condition that its distance $\operatorname{dist}(u)$ is the smallest in set $V_{s}$. Next, $u$ is chosen as the new start point and for every node $v$ in set $V_{s}$, if $\langle u, v\rangle \in L$, and $\operatorname{dis}(u)+w_{u, v}<\operatorname{dis}(v)$, then $\operatorname{dis}(v)=\operatorname{dis}(u)+w(u, v)$ and delete $v$ from set $V_{s}$. Finally, repeat the above process until $V_{s}$ is empty or reaches the finishing point e.

\subsection{Local Path Planning}

After getting the above global optimal path, UGV can drive from the start point to the finishing point according to the path. However, when an obstacle is detected in the global optimal path, the local path planning is needed to help UGV modify the path and avoid the obstacles. To address the above mentioned problem, a local path planning method based on the cost map is proposed in this paper.

The cost map mainly consists of back cost map, lane cost map and obstacle cost map. The back cost map is generated from the global optimal path: the cost of the path is the lowest and the cost increases laterally. The lane cost map is generated from the lane detection results: the cost of the each detected lane is the highest and the cost increases laterally two lanes. The obstacle cost map is generated from the Lidar point cloud data: the traversable area is calculated from the point cloud data and the Gaussian blur operation and dilation operation are conducted to ensure the safety driving of UGV. Then all the above cost map are merged into one cost map and the cost of each lane is calculated as follows;

$$
\text { Total Cost } t^{(i)}=w_{1} \times \text { Back Cos } t+w_{2} \times \sum_{k=1}^{301} \operatorname{Lane} \operatorname{Cos} t^{(i)}\left(x_{k}{ }^{i}, y_{k}{ }^{i}\right)+w_{3} \times \sum_{k=1}^{301} \operatorname{dis}_{k}{ }^{-1} \text { Obstacle Cos } t^{(i)}\left(x_{k}{ }^{i}, y_{k}{ }^{i}\right)
$$

where $w_{1}, w_{2}, w_{3}$ is the weight for back cost, lane cost and obstacle cost respectively. dis $s_{k}$ is the distance between point $\mathrm{k}$ and the vehicle, which is used to give more focus for the near obstacle than the distant obstacle. 


\section{Experiment}

The research centre of intelligent vehicle in Changshu, China is chosen as the test area to validate the effectiveness of the path guiding method proposed in this paper. Figure 5(a) gives the global optimal path, which traverses all the given task point smoothly and gives a reasonable guidance for UGV. The comparison of Figure 5 (b) and (c) shows that the proposed algorithm guides UGV to drive in the turn-right lane when UGV needs to turn right in the next intersection while the tradition Dijkstra algorithm guides UGV to drive in the same lane which violates the traffic rules. Since the proposed algorithm can guide UGV to follow the traffic rules.

To evaluate the effectiveness of the local path planning method, a test scene is placed that two vehicles are randomly put in the global optimal path and the horizontal and lateral distance between two vehicles are $15 \mathrm{~m}$ and $3 \mathrm{~m}$ respectively. In the test scene, UGV need to turn both left and right for one time to pass the obstacles and drive through the test scene. Figure 6(a) gives the global optimal path and the driving path of UGV and from this figure we can see that the cost of the lane that passes the vehicles is larger than other lanes due to the two vehicles ahead. And UGV turns left first and then right to avoid the obstacles with the guidance from the lane cost, which is reasonable for passing the obstacles. Figure 6(b) gives the video sequences of UGV to avoid the vehicles.

Besides the above real scenes experiments, UGV platform with the proposed path guiding algorithm took part in the 2012-2016 Chinese future challenge contest of intelligent vehicles. In the 2016 contest, the UGV platform finished the contest without human intervention and received great results.

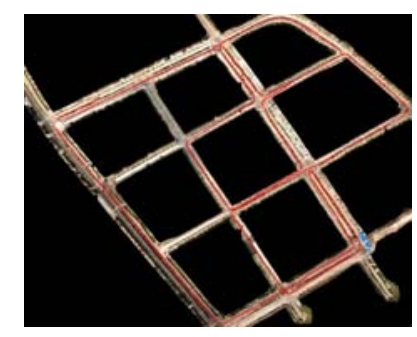

(a)global optimal path
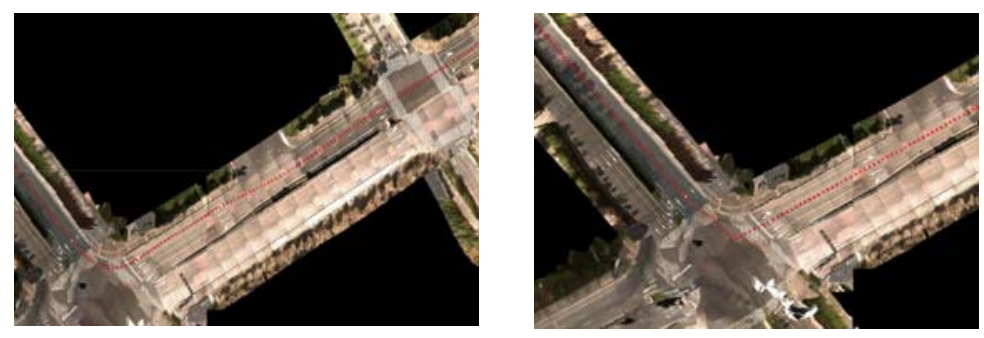

(b) Dijkstra guided path

(c) the proposed algorithm guided path

Figure 5. global path planning results.

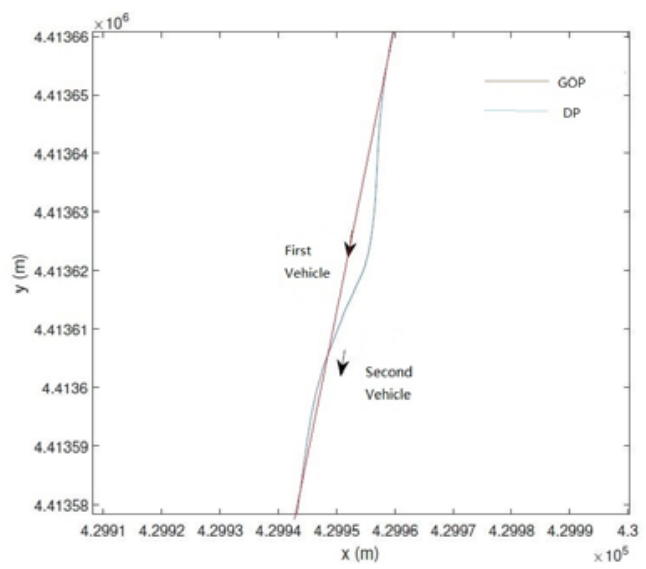

(a) the global optimal path(GOP) and driving path(DP)
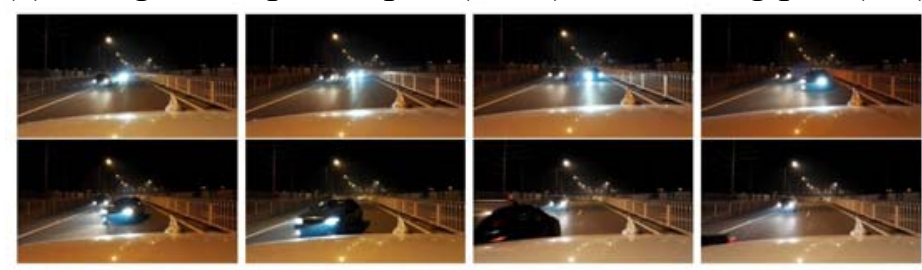

(b) video sequences

Figure 6. Local path planning results. 


\section{Conclusion}

A lane-level digital map is constructed with multi-sensors to meet the requirements of unmanned ground vehicle for high-precision digital map and the needs to navigate in structured road environment. Based on this lane-level digital map, a lane-level path guiding algorithm is also proposed. First, a lane-level digital map is constructed with the multi-sensor data collected by data collecting platform and the lanes and segments are built according to the traffic rules. Next, different property tables are designed for different geographic elements to represent their characteristics. Then, a global path planning method is proposed according to the characteristic of structured road environment. Finally, a local path planning method is proposed to avoid the obstacles when UGV meets the obstacles. According to the results of on-vehicle experiments and contests for unmanned vehicles, the proposed digital map and path guiding method prove to meet the requirements of unmanned ground vehicles in structured road environment and offer reliable guidance and guarantee for fast and safe driving of unmanned ground vehicles.

The high-precision digital map is essential in UGV with the application of UGV in intelligent transportation system and advanced driver assistant system. In this paper, a lane-level digital map is constructed with multi-sensors to meet the requirements of unmanned ground vehicle for highprecision digital map and the needs to navigate in structured road environment. Based on this lanelevel digital map, a lane-level path guiding algorithm is also proposed. From the results of the experiments and the contests, the proposed algorithm meets the requirements of UGV to drive in the unstructured environment and offer reliable guidance to UGV. However, the proposed algorithm is still preliminary research and there are still many problems to be solved. For instance, the line segments of the digital map is still hand-drawn and inefficient, and future work may include that using the multi-sensor data to generate the line segments automatically.

\section{Acknowledgements}

This work was supported by Science and Technology Planning Project of Guangdong Province (2016B090918084).The authors would like to thank the reviewers for their valuable and helpful comments, which have helped improved the paper considerably.

\section{References}

[1]. Zheng B., Yang T., Qiang X.L. Mapping for lane-level digital maps based on mobile mapping systems[J]. Modern surveying and mapping, 2016,39(6):42-45(In Chinses).

[2]. He Y., Lu H., Wang C.X, et.al. Generation of precise lane-level maps based on multi-sensors[J]. Journal of Chang'an University, 2015, 35(1), 274-278.

[3]. Guo C.Z, Kidono K., Junichi M., et.al. A low-cost solution for automatic lane-level map generation using conventional in-car sensors[J]. IEEE Transaction on intelligent transportation system,2016,17(8):2355-2366.

[4]. Zhang H.L., Huang M., Modeling DynasTIM simulation road network based on lane-level basic road network[J]. ACTA SCIENTIARUM NATURALIUM UNIVERSITATIS SUNYATSENI, 2017, 56(1): 53-57.

[5]. Cao G.Y, Damerow F., Flade B., et.al. Camera to map alignment for accurate low-cost lanelevel scene interpretation[C]. 2016 IEEE 19th International Conference on Intelligent Transportation Systems in Rio de Janeiro, Brazil(ITSC), IEEE, 2016:498-504

[6]. Rabe J., Meinke M., Necker M., et.al. Lane-level map-matching based on optimization[C]. 2016 IEEE 19th International Conference on Intelligent Transportation Systems in Rio de Janeiro, Brazil(ITSC), IEEE, 2016:1155-1160.

[7]. Zhang L T, Fu M Y, Yang Y, et al. A Framework of Traffic Lights Detection, Tracking and Recognition Based on Motion Models [C]. 2014 IEEE 17th International Conference on Intelligent Transportation Systems in Qingdao, China (ITSC), IEEE, 2014: 2298 -2303. 